TRANSACTIONS OF THE

AMERICAN MATHEMATICAL SOCIETY

Volume 360, Number 6, June 2008, Pages 2941-2963

S 0002-9947(07)04359-0

Article electronically published on December 11, 2007

\title{
STRONG INTEGRALITY OF QUANTUM INVARIANTS OF 3-MANIFOLDS
}

\author{
THANG T. Q. LE
}

\begin{abstract}
We prove that the quantum $S O(3)$-invariant of an arbitrary 3manifold $M$ is always an algebraic integer if the order of the quantum parameter is co-prime with the order of the torsion part of $H_{1}(M, \mathbb{Z})$. An even stronger integrality, known as cyclotomic integrality, was established by Habiro for integral homology 3-spheres. Here we also generalize Habiro's result to all rational homology 3 -spheres.
\end{abstract}

\section{INTRODUCTION}

0.1 . Integrality at roots of non-prime order. Let $\tau_{M}(q)$ be the quantum $S O(3)$-invariant of a 3 -manifold $M$, which can be defined when $q$ is a complex root of unity of order odd and greater than 1 . The quantum $S U(2)$-invariant was defined by Reshetikhin and Turaev (see [Tur]) and the $S O(3)$-version was defined by Kirby-Melvin [KM] and Turaev. One important result in quantum topology, first proved by $\mathrm{H}$. Murakami $[\mathrm{Mu}]$, is that $\tau_{M}(q) \in \mathbb{Z}[q]$, when the order of $q$ is an odd prime and $M$ a rational homology 3 -sphere. The result was generalized to all 3-manifolds by Masbaum and Roberts [MR], using a short, beautiful proof. Masbaum and Wenzl [MW], and Takata and Yokota [TY] generalized the result to some other Lie algebras, including the $s l_{n}$ series. The author eventually gave a unified proof of the integrality for all Lie algebras [Le3].

The integrality has many important applications; among them is the construction of an integral topological quantum field theory of P. Gilmer and G. Masbaum [GM] and representations of mapping class groups over $\mathbb{Z}$.

In the above integrality, the order of $q$ must be an odd prime, while $\tau_{M}(q)$ can be defined at any root of odd order. Using a quite different method, Habiro [Ha1] showed the integrality of $\tau_{M}$ when $M$ is an integral homology 3-sphere at roots of any order. Thus the restriction on the order of $q$ is removed, but there is a restriction on the manifold: $M$ is an integral homology 3-sphere. Then Habiro and the author proved the integrality of $\tau_{M}$ for integral homology 3-spheres at any root of 1 for all simple Lie algebras [HL].

The first main result of this paper is to establish the integrality of $\tau_{M}$ for all 3 -manifolds with a very minor restriction on the order of the roots of unity.

Received by the editors March 2, 2006.

2000 Mathematics Subject Classification. Primary 57M27; Secondary 57M25.

The author was supported in part by the National Science Foundation. 
Theorem 1. (a) Suppose $\xi$ is a root of unity of order odd, greater than 1, and coprime with the order of the torsion part of $H_{1}(M, \mathbb{Z})$, where $M$ is a closed oriented 3-manifold. Then $\tau_{M}(\xi) \in \mathbb{Z}[\xi]$.

(b) Suppose, in addition, $M$ has 0 first Betti number, and $L$ is a colored, algebraically split link in $M$. Then the quantum invariant $\tau_{M, L}(\xi) \in \mathbb{Z}[\xi]$.

We will recall the definition of $\tau_{M}, \tau_{M, L}$ in section 1 . In the proof of the theorem we will make use of a result of G. Andrews concerning Bailey pairs in the theory of $q$-series.

0.2. Stronger integrality: cyclocomic integrality. We use the standard notation $(a ; q)_{n}$ of $q$-calculus

$$
(a)_{n}=(a ; q)_{n}:=\prod_{i=0}^{n-1}\left(1-a q^{i}\right)
$$

For example, $(q ; q)_{n}=(1-q)\left(1-q^{2}\right) \ldots\left(1-q^{n}\right)$. Define the Habiro ring $\widehat{\Lambda}$ by

$$
\widehat{\Lambda}:=\lim _{\leftarrow n} \mathbb{Z}\left[q^{ \pm 1}\right] /\left((q ; q)_{n}\right) .
$$

Formally, $\widehat{\Lambda}$ is the set of all series of the form

$$
f=\sum_{n=0}^{\infty} f_{n}(q)(1-q)\left(1-q^{2}\right) \ldots\left(1-q^{n}\right), \quad \text { where } \quad f_{n}(q) \in \mathbb{Z}\left[q^{ \pm 1}\right] .
$$

When $q=\xi$, a root of unity, only a finite number of terms in the right hand side are not 0 , hence the right hand side defines a complex number, $\operatorname{denoted}^{b y} \operatorname{ev}_{\xi}(f)$. It is clear that $\operatorname{ev}_{\xi}(f) \in \mathbb{Z}[\xi]$. Thus one can consider every $f \in \widehat{\Lambda}$ as a function with domain the set of roots of unity. It turns out $\widehat{\Lambda}$ has remarkable properties and plays an important role in quantum topology: Habiro [Ha2] showed that every $f \in \widehat{\Lambda}$ has a Taylor expansion $T_{1}(f) \in \mathbb{Z}[[q-1]]$ which uniquely determines $f$. Also, if $f(\xi)=g(\xi)$ at infinitely many roots $\xi$ of prime power orders, then $f=g$ in $\widehat{\Lambda}$. The above properties suggest to consider $\widehat{\Lambda}$ as a class of "analytic functions" with domain the set of roots of unity.

Theorem 2 (Habiro [Ha1]). For every integral homology 3-sphere $M$ there is an invariant $I_{M} \in \widehat{\Lambda}$ such that if $\xi$ is a root of unity of order greater than 1 , then $\operatorname{ev}_{\xi}\left(I_{M}\right)=\tau_{M}(\xi)$.

The integrality of the quantum invariant follows immediate from the theorem, since for every $f \in \widehat{\Lambda}$ and $\xi$ a root of unity, $f(\xi) \in \mathbb{Z}[\xi]$. However, belonging to $\widehat{\Lambda}$ is a much stronger integrality. We call it the cyclotomic integrality. For example, the cyclotomic integrality shows that quantum invariants at infinitely many roots of unity of prime orders determine the values at any other roots of unity. In joint work with Habiro [HL], we generalize Theorem 2 to the case of all simple Lie algebras (but still for integral homology 3 -spheres).

0.3. The case of rational homology 3-spheres. One main result of this paper is to establish cyclotomic integrality for rational homology 3 -spheres. 
For a positive integer $d$ let $A_{d}:=\mathbb{Z}\left[\frac{1}{d}\right]\left[q^{ \pm 1 / d}\right]$ and let $\mathbb{N}_{d}$ be the set of positive integers co-prime with $d$. Denote by $\Phi_{s}(t)$ the $s$-th cyclotomic polynomial. Let $\Lambda_{d} \subset \mathbb{Q}\left(q^{1 / d}\right)$ be the ring obtained from $A_{d}$ by adding the inverses of each $\Phi_{s}\left(q^{1 / d}\right)$, with $s$ not co-prime with $d$ :

$$
\Lambda_{d}:=A_{d}\left[\frac{1}{\Phi_{s}\left(q^{1 / d}\right)}, s \notin \mathbb{N}_{d}\right]
$$

The ring that replaces Habiro's ring in the case of rational homology 3-spheres is

$$
\hat{\Lambda}_{d}:=\lim _{\leftarrow n} \Lambda_{d} /\left((q ; q)_{n}\right) .
$$

Let $U_{d}$ be the set of all complex roots of unity with orders odd, greater than 1 , and co-prime with $d$. The ring $\hat{\Lambda}_{d}$ will play the role of the Habiro ring, with the set of all roots of 1 replaced by $U_{d}$.

Remark 0.1. For technical reasons (in the definition of $S U(2)$ or $S O(3)$ invariants) we exclude the trivial case when the order of the root is 1 .

We first define, for each $\xi \in U_{d}$, the evaluation map $\operatorname{ev}_{\xi}$, which replaces $q$ by $\xi$. Suppose $f \in \mathbb{Q}\left[q^{ \pm 1 / h}\right]$, where $h$ is co-prime with $r$, the order of $\xi$. There exists an integer $b$, unique modulo $r$, such that $\left(\xi^{b}\right)^{h}=\xi$. Then we define

$$
\operatorname{ev}_{\xi} f:=\left.f\right|_{q^{1 / h}=\xi^{b}} .
$$

The definition extends to $\operatorname{ev}_{\xi}: \hat{\Lambda}_{d} \rightarrow \mathbb{C}$, since $\operatorname{ev}_{\xi}\left((q ; q)_{n}\right)=0$ if $n \geq r$. The following is a generalization of Habiro's result.

Theorem 3. Suppose $M$ is a rational homology 3-sphere with $\left|H_{1}(M, \mathbb{Z})\right|=d$, a positive integer. There is an invariant $I_{M} \in \hat{\Lambda}_{d}$ such that if $\xi \in U_{d}$, then $\left(\frac{d}{r}\right) \operatorname{ev}_{\xi}\left(q^{(1-d) / 4} I_{M}\right)$ is the quantum $S O(3)$-invariant of $M$. Here $\left(\frac{d}{r}\right)$ is the Jacobi symbol.

The cyclotomic integrality has many rigidity properties described in the next subsections.

Remark 0.2. (1) The reason we single out the factor $q^{(d-1) / 4}$ is because it might not be in $A_{d}=\mathbb{Z}[1 / d]\left[q^{ \pm 1 / d}\right]$; note that it is always in $\mathbb{Z}\left[q^{ \pm 1 / 2 d}\right]$.

(2) Habiro observed that our proof actually showed that $I_{M} \in \hat{\Lambda}_{d^{\prime}}$, where $d^{\prime}$ is the maximal order of elements of $H_{1}(M, \mathbb{Z})$.

(3) For the special case $d=2$, the result of [BBL] is finer than Theorem 3: In $[\mathrm{BBL}]$, Beliakova, Blanchet, and the author proved that when $d=2$, the quantum $S O(3)$ invariants lie in the ring $\lim _{\leftarrow n} \mathbb{Z}\left[q^{ \pm 1 / 2}\right] /\left(\left(-q ;-q^{-1 / 2}\right)_{2 n}\right)$, which is much smaller than $\hat{\Lambda}_{2}$. The finer structure also allowed us in [BBL] to establish a kind of cyclotomic integrality for quantum invariants associated to spin structures and cohomological classes.

(4) When $d$ is odd, the fact that $I_{M} \in \hat{\Lambda}_{d}$ allows us to define $I_{M}$ at roots of unity of even orders (which were forbidden in the definition of the quantum $S O(3)$ invariant). We will discuss these values in a future publication. 
0.4. Evaluation and Taylor expansion of $\hat{\Lambda}_{d}$. For a subset $\Omega$ of $U_{d}$ let

$$
\operatorname{ev}_{\Omega}: \hat{\Lambda}_{d} \rightarrow \prod_{\xi \in \Omega} \mathbb{C}, \quad \text { defined by } \operatorname{ev}_{\Omega}(f)=\left(\operatorname{ev}_{\xi}(f)\right)_{\xi \in \Omega}
$$

Theorem 4. Suppose $\Omega \subset U_{d}$ contains infinitely many roots of order prime powers. If $f, g \in \hat{\Lambda}_{d}$ such that $\operatorname{ev}_{\xi}(f)=\operatorname{ev}_{\xi}(g)$ for every $\xi \in \Omega$, then $f=g$. In other words, the map $\mathrm{ev}_{\Omega}$ is injective.

We will prove that for every $f \in \hat{\Lambda}_{d}$ and $\xi \in U_{d}$, one has $\operatorname{ev}_{\xi}(f) \in \mathbb{Z}[1 / d, \xi]$. Hence the image of $\operatorname{ev}_{\Omega}$ is in $\prod_{\xi \in \Omega} \mathbb{Z}[1 / d, \xi]$. If $f=I_{M}$ for a rational homology 3 -sphere, then Theorem 1 shows that $\operatorname{ev}_{\xi}(f) \in \mathbb{Z}[\xi]$.

Any element $f \in \Lambda_{d}$, considered as a function of the variable $q^{1 / d}$, is analytic at $q^{1 / d}=1$. The Taylor series of $f$, which is a formal power series in $\left(q^{1 / d}-1\right)$, can be converted into a formal power series in $(q-1)$ by

$$
q^{1 / d}-1=(1+(q-1))^{1 / d}-1=\sum_{n=1}^{\infty}\left(\begin{array}{c}
1 / d \\
n
\end{array}\right)(q-1)^{n} .
$$

Thus we obtained an algebra homomorphism $T_{1}: \Lambda_{d} \rightarrow \mathbb{C}[[q-1]]$, which can easily be extended to $T_{1}: \hat{\Lambda}_{d} \rightarrow \mathbb{C}[[q-1]]$. We call $T_{1}(f)$ the Taylor expansion of $f$ at $q=1$, although in general there is no analytic continuation of $f$ to a neighborhood of 1 .

Theorem 5. (a) For any $f \in \hat{\Lambda}_{d}, T_{1}(f)$ has coefficients in $\mathbb{Z}[1 / d]$, i.e. $T_{1}(f) \in$ $\mathbb{Z}[1 / d][[q-1]]$.

(b) The Taylor expansion map at $1, T_{1}: \hat{\Lambda}_{d} \rightarrow \mathbb{Z}[1 / d][[q-1]]$ is injective. In other words, an element in $\hat{\Lambda}_{d}$ is uniquely determined by its Taylor series.

(c) For a rational homology 3 -sphere $M$, the Taylor expansion $T_{1}\left(q^{(1-d) / 4} I_{M}\right)$ is equal to the Ohtsuki series of $M[\mathrm{Oht} 1]$.

Note that because of the factor $q^{(1-d) / 4}$, the coefficients of the Ohtsuki series in general are in $\mathbb{Z}[1 / 2 d]$. There is also a similar result for Taylor expansion at any root $\xi \in U_{d}$.

The above properties suggests to consider $\hat{\Lambda}_{d}$ as a class of "analytic functions" with domain $U_{d}$.

Remark 0.3. Actually, we will prove, as suggested by Habiro, that $\hat{\Lambda}_{d}$ is isomorphic to a ring already defined by Habiro, defined without any denominators. Then Theorems 4 and 5 part (b) follow easily from [Ha2]. In the original version of the paper, we had a longer proof, which was also adapted from [Ha2].

\subsubsection{Applications.}

Corollary 0.4. Suppose $M$ is a rational homology 3-sphere with $\left|H_{1}(M, \mathbb{Z})\right|=d$.

(a) The Le-Murakami-Ohtsuki (LMO) invariant of $M$ (see [LMO, Le1, Oht2]) determines the quantum $S O(3)$-invariant at any root $\xi \in U_{d}$.

(b) The Ohtsuki series of $M$ has coefficients in $\mathbb{Z}[1 / 2 d]$.

(c) If the order of $\xi \in U_{d}$ is $p^{e}$, where $p$ is an odd prime, then the Taylor series $T_{1}\left(q^{(1-d) / 4} I_{M}\right)$, with $q=\xi$, converges p-adically to $\left(\frac{d}{r}\right) \tau_{M}(\xi)$.

(d) The values of $\tau_{M}$ at an infinite subset of roots of order prime powers in $U_{d}$ determine the value of $\tau_{M}$ at any other root in $U_{d}$. 
Note that part (b) and (c), with $e=1$, were conjectured by R. Lawrence and first proved by Rozansky [Ros] using a quite different method. Here they, together with part (d), are easy consequences of our main results. Part (a), which demonstrates one more universal property [Le1] of the LMO invariant, follows from the fact that the Ohtsuki series is obtained from the LMO invariant by way of the $s l_{2}$-weight system; see [Oht2].

0.5. Plan of the paper. Section 1 is devoted to the definition of quantum invariants, mainly to fix the normalization. In section 2 we review basic facts of Habiro's work on the cyclotomic expansion of the colored Jones polynomial. Proofs of Theorems 3, 4, 5, and their generalizations are given in sections 3,4 , and 5 .

\section{Quantum InVARIAnts}

We will use the following notation for elements in $\mathbb{Z}\left[q^{ \pm 1 / 2}\right]$ :

$$
\{n\}=q^{n / 2}-q^{-n / 2}, \quad\{n\} !=\prod_{i=1}^{n}\{i\}, \quad[n]=\frac{\{n\}}{\{1\}}, \quad\left[\begin{array}{c}
n \\
k
\end{array}\right]=\frac{\{n\} !}{\{k\} !\{n-k\} !} .
$$

All 3-manifolds in this paper are supposed to be closed and oriented. Every link in a 3-manifold is a framed, oriented, and has components ordered.

1.1. The colored Jones polynomial. Suppose $L$ is a framed, oriented link in $S^{3}$ with $m$ ordered components. For finite-dimensional $s l_{2}$-modules $W_{1}, \ldots, W_{m}$, one can define the quantum invariant $J_{L}\left(W_{1}, \ldots, W_{m}\right) \in \mathbb{Z}\left[q^{ \pm 1 / 4}\right]$; see [Tur, KM, Oht2]. The modules $W_{1}, \ldots, W_{m}$ are usually called the colors of the link $L$.

It is known that for every positive integer $n$ there is a unique irreducible $s l_{2}$ module $V_{n}$ of dimension $n$. For positive integers $n_{1}, \ldots, n_{m}$ we define $J_{L}\left(n_{1}, \ldots, n_{m}\right)$ $:=J_{L}\left(V_{n_{1}}, \ldots, V_{n_{m}}\right)$.

We recall here a few well-known formulas, and at the same time fix our normalization. For the unknot $U$ with 0 framing one has

$$
J_{U}(n)=[n]=\{n\} /\{1\} .
$$

If $L^{\prime}$ is obtained from $L$ by increasing the framing of the $i$-th component by 1 , then

$$
J_{L^{\prime}}\left(n_{1}, \ldots, n_{m}\right)=q^{\left(n_{i}^{2}-1\right) / 4} J_{L}\left(n_{1}, \ldots, n_{m}\right) .
$$

When all the $n_{i}$ 's are equal to 2 , then $J_{L}\left(n_{1}, \ldots, n_{m}\right)=\tilde{J}_{L} \in \mathbb{Z}\left[q^{1 / 4}\right]$, a version of the Jones polynomial [Jo, Oht2], which satisfies the skein relation:

$$
q^{1 / 4} \tilde{J}_{L^{+}}-q^{-1 / 4} \tilde{J}_{L^{-}}=\left(q^{1 / 2}-q^{-1 / 2}\right) \tilde{J}_{L^{0}},
$$

where $L^{+}, L^{-}, L^{0}$ have a blackboard link diagram identical everywhere, except for a small ball in which $L_{+}$has a positive crossing, $L_{-}$a negative crossing, and $L^{0}$ a resolution of the crossing.

In general, $J_{L}\left(n_{1}, \ldots, n_{m}\right) \in \mathbb{Z}\left[q^{ \pm 1 / 4}\right]$. However, there is a number $a \in\left\{0, \frac{1}{4}, \frac{1}{2}, \frac{3}{4}\right\}$ such that $J_{L}\left(n_{1}, \ldots, n_{m}\right) \in q^{a} \mathbb{Z}\left[q^{ \pm 1}\right]$. For a formula for $a$ and more general results (for all simple Lie algebra), see [Le2]. A special case is the following. 
Lemma 1.1. Suppose L has 0 linking matrix. Then

$$
J_{L}\left(n_{1}, \ldots, n_{m}\right) \in q^{\left(n_{1}+\ldots n_{m}-m\right) / 2} \mathbb{Z}\left[q^{ \pm 1}\right] .
$$

Let $\mathbf{R}$ be the Grothendieck ring of finite-dimensional $s l_{2}$-modules, tensored by $\mathbb{C}\left(q^{1 / 4}\right)$. As a vector space over $\mathbb{C}\left(q^{1 / 4}\right), \mathbf{R}$ is freely spanned by $V_{1}, V_{2}, \ldots$, but as an algebra, it is isomorphic to $\mathbb{C}\left(q^{1 / 4}\right)\left[V_{2}\right]$. Using linearity we can define, for $W_{i} \in \mathbf{R}$, $J_{L}\left(W_{1}, \ldots, W_{m}\right) \in \mathbb{C}\left(q^{1 / 4}\right)$. Thus $J_{L}$ is considered as a multi-linear function from $\mathbf{R}^{m}$ to $\mathbb{C}\left(q^{1 / 4}\right)$.

1.2. Definition of $S O(3)$-invariant of 3-manifolds. Suppose $\xi$ is a root of unity of order $r$, which is odd and greater than 1 , and $f\left(q ; n_{1}, \ldots, n_{m}\right)$ a function of variables $q$ and integers $n_{1}, \ldots, n_{m}$. Let

$$
\sum_{n_{i}}^{\xi} f:=\sum_{n_{i}} \operatorname{ev}_{\xi}(f)
$$

where in the sum all the $n_{i}$ run the set of odd numbers between 0 and $2 r$. Let

$$
F_{L}(\xi):=\sum_{n_{i}}^{\xi}\left(J_{L}\left(n_{1}, \ldots, n_{m}\right) \prod_{i=1}^{m}\left[n_{i}\right]\right) .
$$

Let $U^{ \pm}$be the unknot with framing \pm 1 . It is known that $F_{U^{ \pm}}(\xi) \neq 0$; see Lemma 1.3.

Suppose $M$ is an oriented 3-manifold obtained from $S^{3}$ by surgery along a framed, oriented link $L$. (Note that $M$ does not depend on the orientation of $L$.) Let $\sigma_{+}$ (resp., $\sigma_{-}$) be the number of positive (resp. negative) eigenvalues of the linking matrix of $L$. Suppose $\xi$ is a root of unity of odd order $r$. Then the quantum $S O(3)$-invariant is defined by

$$
\tau_{M}(\xi)=\tau_{M}^{S O(3)}(\xi):=\frac{F_{L}(\xi)}{\left(F_{U^{+}}(\xi)\right)^{\sigma_{+}}\left(F_{U^{-}}(\xi)\right)^{\sigma_{-}}} .
$$

Suppose in addition to $L$, in $S^{3}$ there is another framed link $L^{\prime}$ with $s$ components, and surgery along $L$ transforms $\left(S^{3}, L^{\prime}\right)$ to $\left(M, L^{\prime \prime}\right)$. Then the quantum $S O(3)$-invariant of $\left(M, L^{\prime \prime}\right)$ is defined by

$$
\tau_{M, L^{\prime \prime}}\left(k_{1}, \ldots, k_{s} ; \xi\right):=\frac{\sum_{n_{i}}^{\xi}\left(J_{L \cup L^{\prime}}\left(n_{1}, \ldots, n_{m}, k_{1}, \ldots, k_{s}\right) \prod_{i=1}^{m}\left[n_{i}\right]\right)}{\left(F_{U^{+}}(\xi)\right)^{\sigma_{+}}\left(F_{U^{-}}(\xi)\right)^{\sigma_{-}}} .
$$

For a connected sum, one has $\tau_{M \# N}(\xi)=\tau_{M}(\xi) \tau_{N}(\xi)$.

1.3. Gauss sum, Laplace transform, and the value of $F_{U^{ \pm}}(\xi)$. Suppose that $\xi \in U_{d}$ has odd order $r$. A variation $\gamma_{d}(\xi)$ of the Gauss sum is defined by

$$
\gamma_{d}(\xi):=\sum_{n}^{\xi} q^{d \frac{n^{2}-1}{4}}
$$

It is known that $\left|\gamma_{d}(\xi)\right|=\sqrt{r}$, and hence is never 0 . 
Let $\mathcal{L}_{d ; n}: \mathbb{Z}\left[q^{ \pm n}, q^{ \pm 1}\right] \rightarrow \mathbb{Z}\left[q^{ \pm 1 / d}\right]$ be the $\mathbb{Z}\left[q^{ \pm 1}\right]$-linear operator, called the Laplace transform, defined by

$$
\mathcal{L}_{d ; n}\left(q^{n a}\right):=q^{-a^{2} / d} .
$$

Lemma 1.2. Suppose $\xi \in U_{d}$ is a root of 1 of order $r$ and $f \in \mathbb{Z}\left[q^{ \pm n}, q^{ \pm 1}\right]$. Then

$$
\sum_{n}^{\xi} q^{d \frac{n^{2}-1}{4}} f=\gamma_{d}(\xi) \operatorname{ev} \xi\left(\mathcal{L}_{d ; n}(f)\right) .
$$

Proof. It's enough to consider the case when $f=q^{n a}$, with $a$ an integer. This case is proved simply by using the standard completing the square method; see for example, [Le3].

The point is that $\mathcal{L}_{d ; n}(f)$, unlike the left hand side $\sum_{n}^{\xi} q^{d \frac{n^{2}-1}{4}} f$, does not depend on $\xi \in U_{d}$, and will help us to define a "universal invariant". Applying Lemma 1.2 with $d= \pm 1$ and $f=[n]^{2}$, using the unknot formula (1), we get the following.

Lemma 1.3. For the unknot $U^{ \pm}$with framing \pm 1 , one has $F_{U^{ \pm}}(\xi) \neq 0$. Moreover,

$$
F_{U^{ \pm}}(\xi)=\mp 2 \gamma_{ \pm 1}(\xi) \operatorname{ev}_{\xi}\left(\frac{q^{\mp 1 / 2}}{\{1\}}\right) \text {. }
$$

1.4. Lens spaces. Let $\left(\frac{d}{r}\right)$ be the Jacobi symbol and $s(d, a)$ the Dedekind sum. Recall that

$$
s(d, a):=\sum_{i=1}^{|d|-1}\left(\left(\frac{i}{d}\right)\right)\left(\left(\frac{i a}{d}\right)\right), \quad \text { where }((x)):=x-\lfloor x\rfloor-1 / 2 .
$$

For co-prime integers $a, d$ with $d>0$, the $S O(3)$-invariant of the lens space $L(d, a)$, which is obtained by surgery along the unknot with rational framing $d / a$, is given by (see $[\mathrm{LL}])$

$$
\tau_{L(d, a)}(\xi)=\left(\frac{d}{r}\right) \operatorname{ev} \xi\left(q^{-3 s(d, a)} \frac{q^{1 / 2 d}-q^{-1 / 2 d}}{q^{1 / 2}-q^{-1 / 2}}\right) .
$$

In particular, $\tau_{L(d, a)}(\xi)$ is invertible in $\mathbb{Z}[\xi]$.

It is also well-known that for every non-zero integer $d$, if $\operatorname{sn}(d)$ is the sign of $d$, then

$$
\frac{\gamma_{d}(\xi)}{\gamma_{\mathrm{sn}(d)}(\xi)}=\left(\frac{|d|}{r}\right) \operatorname{ev}_{\xi}\left(q^{(\operatorname{sn}(d)-d) / 4}\right)
$$

\section{HABIRo's CyClotomic EXPANSION OF THE COLORED JONES POLYNOMIAL}

2.1. The basis $P_{n}^{\prime}$. Recall that $\mathbf{R}$ is isomorphic to $\mathbb{C}\left(q^{1 / 4}\right)\left[V_{2}\right]$, with $V_{1}, V_{2}, \ldots$ as a basis over $\mathbb{C}\left(q^{1 / 4}\right)$. Habiro [Ha1] defined a new basis $P_{k}^{\prime}, k=0,1,2, \ldots$, where

$$
P_{k}^{\prime}:=\frac{1}{\{k\} !} \prod_{i=1}^{k}\left(V_{2}-q^{(2 i-1) / 2}-q^{-(2 i-1) / 2}\right) .
$$

The change from the basis $V_{n}$ to $P_{k}^{\prime}$ is given by

$$
V_{n}=\sum_{k=0}^{n-1}\left[\begin{array}{c}
n+k \\
2 k+1
\end{array}\right]\{k\} ! P_{k}^{\prime},
$$


where the sum over $k$ can be made from 0 to infinity, since $\left[\begin{array}{c}n+k \\ 2 k+1\end{array}\right]=0$ if $k \geq n$.

For any link $L$, using the linearity of $J_{L}$, one has

$$
J_{L}\left(n_{1}, \ldots, n_{m}\right)=\sum_{0 \leq k_{i} \leq n_{i}-1} J_{L}\left(P_{k_{1}}^{\prime}, \ldots, P_{k_{m}}^{\prime}\right) \prod_{i=1}^{m}\left[\begin{array}{c}
n_{i}+k_{i} \\
2 k_{i}+1
\end{array}\right]\left\{k_{i}\right\} !
$$

Since there is a denominator in the definition of $P_{k}^{\prime}$, one might expect that $J_{L}\left(P_{k_{1}}^{\prime}, \ldots, P_{k_{m}}^{\prime}\right)$ also has a non-trivial denominator. A difficult and important integrality result of Habiro [Ha1] is

Theorem 6 ([Ha1, Thm.3.3]). If $L$ is algebraically split and zero framed link in $S^{3}$, then

$$
J_{L}\left(P_{k_{1}}^{\prime}, \ldots, P_{k_{m}}^{\prime}\right) \in \frac{\{2 k+1\} !}{\{k\} !\{1\}} \mathbb{Z}\left[q^{ \pm 1 / 2}\right]=\left[\begin{array}{c}
2 k+1 \\
k
\end{array}\right]\left(q^{2}\right)_{k} \mathbb{Z}\left[q^{ \pm 1 / 2}\right],
$$

where $k=\max \left\{k_{1}, \ldots, k_{m}\right\}$.

Thus, $J_{L}\left(P_{k_{1}}^{\prime}, \ldots, P_{k_{m}}^{\prime}\right)$ is not only integral, but also divisible by $\left[\begin{array}{c}2 k+1 \\ k\end{array}\right]\left(q^{2}\right)_{k}$, which, in turn, is divisible by $(q)_{k}$.

\subsection{Evaluation at $\xi$.}

Lemma 2.1. Suppose $\xi$ is a root of unity whose order $r$ is odd and greater than 2 . If $k>(r-3) / 2$, then

$$
\operatorname{ev}_{\xi}\left(\frac{\{2 k+1\} !}{\{k\} !\{1\}}\right)=0
$$

Proof. First assume that $k \geq r-1$. Note that

$$
\frac{\{2 k+1\} !}{\{k\} !\{1\}}=\left[\begin{array}{c}
2 k+1 \\
k
\end{array}\right]\{2\}\{3\} \ldots\{k+1\} .
$$

Each factor of the right hand side is polynomial in $q^{1 / 2}$, and $r$ is among $\{2,3, \ldots$, $k+1\}$. Since $\operatorname{ev}_{\xi}\{r\}=0$, the evaluation $\mathrm{ev}_{\xi}$ of the left hand side is 0 .

It remains to consider the case $k+1<r \leq 2 k+1$. Note that

$$
\frac{\{2 k+1\} !}{\{k\} !\{1\}}=\frac{\{k+1\}\{k+2\} \ldots\{2 k+1\}}{\{1\}} .
$$

When evaluating using $\mathrm{ev}_{\xi}$, the denominator is not 0 , but the numerator is, since $r$ is among $\{k+1, k+2, \ldots, 2 k+1\}$.

From the above lemma and Theorem 6 we have the following.

Corollary 2.2. Suppose $\xi$ is a root of unity of odd order $r>2$ and $L$ an algebraically split link with 0-framing on each component. Then

$$
\operatorname{ev}_{\xi}\left(J_{L}\left(n_{1}, \ldots, n_{m}\right)\right)=\operatorname{ev}_{\xi}\left(\sum_{k_{1}, \ldots, k_{m}=0}^{(r-3) / 2} J_{L}\left(P_{k_{1}}^{\prime}, \ldots, P_{k_{m}}^{\prime}\right) \prod_{i=1}^{m}\left[\begin{array}{c}
n_{i}+k_{i} \\
2 k_{i}+1
\end{array}\right]\left\{k_{i}\right\} !\right) .
$$




\section{INTEGRALITY OF QUANTUM INVARIANTS}

\subsection{Technical results.}

\subsubsection{Divisibility of the Laplace transform images.}

Proposition 3.1. Suppose $\xi \in U_{d}$ is a root of order odd $r$ and $k \leq(r-3) / 2$. Choose an integer $b$ such that $d b \equiv 1(\bmod r)$. Then

$$
\begin{aligned}
\sum_{n}^{\xi}\left[\begin{array}{c}
n+k \\
2 k+1
\end{array}\right]\{k\} !\{n\} & =2 \operatorname{ev} \xi\left(q^{(k+1)(k+2) / 4}\left(q^{k+2}\right)_{r-k-2}\right), \\
\sum_{n}^{\xi} q^{d \frac{n^{2}-1}{4}}\left[\begin{array}{c}
n+k \\
2 k+1
\end{array}\right]\{k\} !\{n\} & =-2 \operatorname{sn}(b) \gamma_{d}(\xi) \operatorname{ev}_{\xi}(H(k,-b)),
\end{aligned}
$$

where $H(k,-b)$ is in $\mathbb{Z}\left[q^{ \pm 1 / 2}\right]$.

The proof will be given later in this section. The upshot here is that the right hand side of (6) is divisible by $\gamma_{d}(\xi)$. An explicit formula for $H(k, b)$, defined for any pairs $(k, b)$ of non-zero integers and not depending on $\xi$, as well as the proof of the proposition, is given in section 3.4.

3.1.2. Diagonalizing the linking matrix. A link $L$ in a 3-manifold $M$ is algebraically split if each component of $L$ bounds an orientable surface which does not intersect any other component.

Proposition 3.2. (a) Suppose $M$ is a 3-manifold with $\left|\operatorname{Tor}\left(H_{1}(M, \mathbb{Z})\right)\right|=d$, with prime decomposition $d=p_{1}^{e_{1}} \ldots p_{k}^{e_{k}}$. Then there are lens spaces $L\left(d_{i}, a_{i}\right)$ such that each $d_{i}$ is a power of one of the $p_{j}$, and the connected sum of $M$ and these lens spaces can be obtained by integral surgery along an algebraically split link in $S^{3}$.

(b) Suppose in addition $M$ is a rational homology 3-sphere, i.e. $\left|H_{1}(M, \mathbb{Z})\right|=d$. Assume that $L$ is an algebraically split link in $M$. Then there is an algebraically split link $\bar{L}$, which is the disjoint union of 2 sub-links $\bar{L}_{1}$ and $\bar{L}_{2}$, in $S^{3}$ such that surgery along $\bar{L}_{1}$ transforms $\left(S^{3}, \bar{L}_{2}\right)$ to $\left(M^{\prime}, L\right)$. Here $M^{\prime}$ is the connected sum of $M$ and several lens spaces $L\left(d_{i}, a_{i}\right)$ with properties as described in part (a).

The proof will be given later. The proposition, part (a), with $M$ a rational homology 3-sphere, is a modification of Ohtsuki's lemma [Oht1].

3.2. The case of a diagonal linking matrix. Suppose $M^{3}$ is obtained from $S^{3}$ by surgery along an algebraically split $(m+s)$-component link $L$ with integral framings $d_{1}, \ldots, d_{m+s}$ on the link components, where $d_{m+1}=\cdots=d_{m+s}=0$ and all other $d_{i}$ 's are not 0 . Let $L^{0}$ denote the link $L$ with all framings switched to 0 . Let $d=\prod_{i=1}^{m}\left|d_{i}\right|$. Suppose $\xi \in U_{d}$ is a root of unity of odd order $r$. Choose integer $b_{i}$ such that $b_{i} d_{i}>0$ and $b_{i} d_{i}=1(\bmod r)$, for $i=1, \ldots, m$.

3.2.1. Non-degenerate diagonal linking matrix. First we consider the case when $s=0$, i.e. the linking matrix is non-degenerate, or $M$ is a rational homology 3 -sphere. 
By framing formula (2),

$$
\begin{aligned}
J_{L}\left(n_{1}, \ldots, n_{m}\right) & =J_{L^{0}}\left(n_{1}, \ldots, n_{m}\right) \prod_{i=1}^{m} q^{d_{i} \frac{n_{i}^{2}-1}{4}}, \quad \text { and hence by formula }(2.2) \\
\operatorname{ev}_{\xi}\left(J_{L}\left(n_{1}, \ldots, n_{m}\right)\right) & =\operatorname{ev} \xi\left(\sum_{k_{i}=0}^{(r-3) / 2} J_{L^{0}}\left(P_{k_{1}}^{\prime}, \ldots, P_{k_{1}}^{\prime}\right) \prod_{i=1}^{m} q^{d_{i} \frac{n_{i}^{2}-1}{4}}\left[\begin{array}{c}
n_{i}+k_{i} \\
2 k_{i}+1
\end{array}\right]\left\{k_{i}\right\} !\right) .
\end{aligned}
$$

By definition,

$$
F_{L}(\xi)=\sum_{n_{i}}^{\xi}\left(J_{L}\left(n_{1}, \ldots, n_{m}\right) \prod_{i=1}^{m}\left[n_{i}\right]\right), \quad \text { and hence }
$$

$$
\begin{aligned}
F_{L}(\xi) & =\operatorname{ev} \xi\left(\sum_{k_{i}=0}^{(r-3) / 2} J_{L^{0}}\left(P_{k_{1}}^{\prime}, \ldots, P_{k_{m}}^{\prime}\right) \prod_{i=1}^{m} \sum_{n_{i}}^{\xi} q^{d_{i} \frac{n_{i}^{2}-1}{4}}\left[\begin{array}{c}
n_{i}+k_{i} \\
2 k_{i}+1
\end{array}\right] \frac{\left\{k_{i}\right\} !\left\{n_{i}\right\}}{\{1\}}\right) \\
8) & =\left(\prod_{i=1}^{m} \frac{-2 \operatorname{sn}\left(d_{i}\right) \gamma_{d_{i}}(\xi)}{\operatorname{ev}_{\xi}\{1\}}\right) \operatorname{ev} \xi\left(\sum_{k_{i}=0}^{(r-3) / 2} J_{L^{0}}\left(P_{k_{1}}^{\prime}, \ldots, P_{k_{m}}^{\prime}\right) \prod_{i=1}^{m} H\left(k_{i},-b_{i}\right)\right)
\end{aligned}
$$

by $(6)$.

Using Lemma 1.3 and the definition of $\tau_{M}$, one obtains

$$
\tau_{M}(\xi)=\prod_{i=1}^{m} \frac{\gamma_{d_{i}}(\xi)}{\gamma_{\mathrm{sn}\left(d_{i}\right)}(\xi)} \operatorname{ev} \xi\left(\sum_{k_{i}=0}^{(r-3) / 2} J_{L^{0}}\left(P_{k_{1}}^{\prime}, \ldots, P_{k_{m}}^{\prime}\right) \prod_{i=1}^{m} q^{\mathrm{sn}\left(d_{i}\right) 1 / 2} H\left(k_{i},-b_{i}\right)\right)
$$

Using (4) for $\frac{\gamma_{d_{i}}(\xi)}{\gamma_{\mathrm{sn}\left(d_{i}\right)}(\xi)}$, we get

(9) $\tau_{M}(\xi)=\left(\frac{d}{r}\right) \operatorname{ev} \xi\left(\sum_{k_{i}=0}^{(r-3) / 2} J_{L^{0}}\left(P_{k_{1}}^{\prime}, \ldots, P_{k_{m}}^{\prime}\right) \prod_{i=1}^{m} q^{\left(3 \operatorname{sn}\left(d_{i}\right)-d_{i}\right) / 4} H\left(k_{i},-b_{i}\right)\right)$.

3.2.2. Degenerate diagonal linking matrix. Now we assume the general case, where $s$ might not be 0 .

The argument that leads to (7) will give us

$$
\begin{array}{r}
F_{L}(\xi)=\sum_{k_{i}=0}^{(r-3) / 2} \operatorname{ev}_{\xi}\left(J_{L^{0}}\left(P_{k_{1}}^{\prime}, \ldots, P_{k_{m+s}}^{\prime}\right) \prod_{i=1}^{m} \sum_{n_{i}}^{\xi} q^{d_{i} \frac{n_{i}^{2}-1}{4}}\left[\begin{array}{c}
n_{i}+k_{i} \\
2 k_{i}+1
\end{array}\right] \frac{\left\{k_{i}\right\} !\left\{n_{i}\right\}}{\{1\}}\right) \\
\times\left(\prod_{i=m+1}^{m+s} \sum_{n_{i}}^{\xi}\left[\begin{array}{c}
n_{i}+k_{i} \\
2 k_{i}+1
\end{array}\right] \frac{\left\{k_{i}\right\} !\left\{n_{i}\right\}}{\{1\}}\right) .
\end{array}
$$


Using Proposition 3.1, we get

(10)

$$
\begin{array}{r}
\tau_{M}(\xi)=\left(\frac{d}{r}\right) \sum_{k_{i}=0}^{(r-3) / 2} \operatorname{ev} \xi\left(J_{L^{0}}\left(P_{k_{1}}^{\prime}, \ldots, P_{k_{m+s}}^{\prime}\right) \prod_{i=1}^{m} q^{\left(3 \operatorname{sn}\left(d_{i}\right)-d_{i}\right) / 4} H\left(k_{i},-b_{i}\right)\right) \\
\times \operatorname{ev}_{\xi}\left(\prod_{i=m+1}^{m+s} 2 q^{\left(k_{i}+1\right)\left(k_{i}+2\right) / 4} \frac{\left(q^{k_{i}+2}\right)_{r-k_{i}-2}}{\{1\}}\right) .
\end{array}
$$

Note that, since $k_{i} \leq(r-3) / 2$, one has $\frac{\left(q^{k_{i}+2}\right)_{r-k_{i}-2}}{\{1\}} \in \mathbb{Z}\left[q^{ \pm 1 / 2}\right]$. It follows that $\tau_{M}(\xi) \in \mathbb{Z}[\xi]$.

3.3. Proof of Theorem 1. Part (a). By the product formula, $\tau_{M}=\tau_{(M \# N)} / \tau_{N}$, if $\tau_{N} \neq 0$. Since $\tau_{M}(\xi)$ (with $M$ the lens space $L(d, a)$ and $\xi \in U_{d}$ ) is invertible in $\mathbb{Z}[\xi]$, using Proposition 3.2 we can assume that $M$ is obtained from a link with a diagonal linking matrix as described in the previous subsection. There we have proved that $\tau_{M}(\xi) \in \mathbb{Z}[\xi]$.

Part (b). Again we can assume that $(M, L)$ is obtained from $\left(S^{3}, \bar{L}_{2}\right)$ by surgery along $\bar{L}_{1}$, as described in Proposition 3.2.

Similarly to (10) we have

$$
\begin{aligned}
\tau_{M, L}\left(n_{m+1}, \ldots, n_{m+s}\right) & \\
=\left(\frac{d}{r}\right) \operatorname{ev} \xi & \left(\sum_{k_{i}=0}^{(r-3) / 2} J_{\bar{L}^{0}}\left(P_{k_{1}}^{\prime}, \ldots, P_{k_{m+s}}^{\prime}\right) \prod_{i=1}^{m} q^{\left(3 \operatorname{sn}\left(d_{i}\right)-d_{i}\right) / 4} H\left(k_{i},-b_{i}\right)\right) \\
& \times \operatorname{ev} \xi\left(\prod_{i=m+1}^{m+s}\left(\begin{array}{c}
n_{i}+k_{i} \\
2 k_{i}+1
\end{array}\right)\left\{k_{i}\right\} !\right)
\end{aligned}
$$

which is in $\mathbb{Z}[\xi]$.

3.4. Proofs of technical results. For a non-negative integer $k$ let

$$
Z(k):=\sum_{j=0}^{2 k+1}(-1)^{j}\left[\begin{array}{c}
2 k+1 \\
j
\end{array}\right] t^{(j-k)^{2}} \in \mathbb{Z}\left[q^{ \pm 1}, t^{ \pm 1}\right] .
$$

For an integer $d \neq 0$ and an arbitrary integer $b$ define the $\mathbb{Z}\left[q^{ \pm 1}\right]$-linear algebra operators

$$
\begin{aligned}
\varphi_{d}: \mathbb{Z}\left[q^{ \pm 1}, t^{ \pm 1}\right] \rightarrow \mathbb{Z}\left[q^{ \pm 1 / d}\right], & & \text { where } \varphi_{d}(t):=q^{1 / d} \\
\tilde{\varphi}_{b}: \mathbb{Z}\left[q^{ \pm 1}, t^{ \pm 1}\right] \rightarrow \mathbb{Z}\left[q^{ \pm 1}\right], & & \text { where } \tilde{\varphi}_{b}(t)=q^{b}
\end{aligned}
$$

The relation between $\varphi$ and $\tilde{\varphi}$ is: If $\xi \in U_{d}$ has order $r$, and $d b \equiv 1(\bmod r)$, then for every $f \in \mathbb{Z}\left[q^{ \pm 1}, t^{ \pm 1}\right]$ :

$$
\operatorname{ev}_{\xi}\left(\varphi_{d}(f)\right)=\operatorname{ev}_{\xi}\left(\tilde{\varphi}_{b}(f)\right)
$$


For a non-zero integer $d$ and an arbitrary integer $b$ let us define

$$
\begin{aligned}
Y(k, d) & :=\varphi_{d}(Z(k))=\sum_{j=0}^{2 k+1}(-1)^{j}\left[\begin{array}{c}
2 k+1 \\
j
\end{array}\right] q^{(j-k)^{2} / d}, \\
\tilde{Y}(k, b) & :=\tilde{\varphi}_{b}(Z(k))=\sum_{j=0}^{2 k+1}(-1)^{j}\left[\begin{array}{c}
2 k+1 \\
j
\end{array}\right] q^{b(j-k)^{2}} .
\end{aligned}
$$

Lemma 3.3. Suppose $b d \equiv 1(\bmod r)$, where $r$ is the order of $\xi \in U_{d}$; then

$$
\sum_{n}^{\xi} q^{d \frac{n^{2}-1}{4}}\left[\begin{array}{c}
n+k \\
2 k+1
\end{array}\right]\{k\} !\{n\}=-2 \gamma_{d}(\xi) \operatorname{ev}_{\xi}\left(\frac{\tilde{Y}(k,-b)\{k\} !}{\{2 k+1\} !}\right) .
$$

Proof. We will first calculate $\mathcal{L}_{d ; n}(\{n\}\{n+k\} ! /\{n-k-1\} !)$.

Using $\{j\}=-q^{-j / 2}\left(1-q^{j}\right)$, one sees that

$$
\begin{aligned}
\{n\}\{n+k\} ! /\{n-k-1\} ! & =q^{-n k}\left(q^{-n}-1\right)\left(q^{n-k} ; q\right)_{2 k+1} \\
& =q^{-n-n k}\left(q^{n-k} ; q\right)_{2 k+1}-q^{-n k}\left(q^{n-k} ; q\right)_{2 k+1} .
\end{aligned}
$$

It is easy to check that the two terms of the right hand side of (12) can be obtained from one another by the involution $n \rightarrow-n$. Since $\mathcal{L}_{d ; n}$ is invariant under $n \rightarrow-n$, one has

$$
\mathcal{L}_{d ; n}(\{n\}\{n+k\} ! /\{n-k-1\} !)=-2 \mathcal{L}_{d ; n}\left(q^{-n k}\left(q^{n-k} ; q\right)_{2 k+1}\right) .
$$

Explicit expansion of $(a ; q)_{k}$ is well-known, and we have

$$
q^{-n k}\left(q^{n-k} ; q\right)_{2 k+1}=\sum_{j=0}^{2 k+1}(-1)^{j}\left[\begin{array}{c}
2 k+1 \\
j
\end{array}\right] q^{n(j-k)} .
$$

Using the definition of $\mathcal{L}_{d ; n}$ and (13) we get

$$
\mathcal{L}_{d ; n}(\{n\}\{n+k\} ! /\{n-k-1\} !)=-2 Y(k,-d) .
$$

Multiplying by $\{k\} ! /\{2 k+1\}$ ! and using Lemma 1.2 and (11), we get the lemma.

3.4.1. Factoring $\tilde{Y}(k, b)$. It turns out that $\tilde{Y}(k, b)$ is always divisible by $\frac{\{2 k+1\} !}{\{k\} !}$. To describe the quotient let us define, for a positive integer $b$,

$$
H(k, b):=q^{(k+1)(k+2) / 4} \sum_{k+1 \geq n_{b} \geq n_{b-1} \geq \cdots \geq n_{2} \geq 0} q^{n_{2}^{2}+n_{3}^{2}+\cdots+n_{b}^{2}} \frac{(q)_{k+1}}{\prod_{i=1}^{b}(q)_{n_{i+1}-n_{i}}},
$$

where $n_{b+1}=k+1$ and $n_{1}=0$, and $(a)_{n}$ stands for $(a ; q)_{n}$. For example,

$$
\begin{aligned}
& H(k, 1)=q^{(k+1)(k+2) / 4} \\
& H(k, 2)=q^{(k+1)(k+2) / 4} \sum_{j=0}^{k+1} q^{j^{2}} \frac{(q)_{k+1}}{(q)_{j}(q)_{k+1-j}}, \\
& H(k, 3)=q^{(k+1)(k+2) / 4} \sum_{0 \leq j \leq l \leq k+1} q^{j^{2}+l^{2}} \frac{(q)_{k+1}}{(q)_{j}(q)_{l-j}(q)_{k+1-l}} .
\end{aligned}
$$

Let $\overline{H(k, b)}$ be obtained from $H(k, b)$ by the involution $q \rightarrow q^{-1}$. For $b=0$ define $H(k, b)=0$ and for $b<0$ let

$$
H(k, b):=(-1)^{k} \overline{H(k,-b)} .
$$


Remark 3.4. Habiro observed there is a similarity between $H(k, b)$ and the coefficient $c_{n, p}^{\prime}$ of Habiro's twists in [Ma]. We have found the exact relation and will discuss it in another publication.

The following theorem is the main technical result.

Theorem 7. For every integer $b, \tilde{Y}(k, b)$ is divisible by $\frac{\{2 k+1\} !}{\{k\} !}$. Moreover

$$
\tilde{Y}(k, b) \frac{\{k\} !}{\{2 k+1\} !}=-\operatorname{sn}(b) H(k, b) .
$$

Proof. If $b=0$, then $\tilde{Y}(k, b)$ is the right hand side of $(14)$, with $n=0$. In this case (with $n=0$ ), the left hand side of (14) is 0 . Hence $\tilde{Y}(k, 0)=0$, and we are done.

Suppose $b \neq 0$. Using the involution $q \rightarrow q^{-1}$, we can assume that $b>0$.

Let $\alpha_{n}, \beta_{n}$ be a Bailey pair as defined in Section 3.4 of [An], with $a=1$. Then for any numbers $b_{i}, c_{i}, i=1, \ldots, k$, and a positive integer $N$ we have the identity (3.43) of $[\mathrm{An}]$ :

$$
\begin{aligned}
\sum_{n \geq 0}(-1)^{n} \alpha_{n} q^{-\left(\begin{array}{c}
n \\
2
\end{array}\right)+k n+N n} \frac{\left(q^{-N}\right)_{n}}{\left(q^{N+1}\right)_{n}} \prod_{i=1}^{k} \frac{\left(b_{i}\right)_{n}}{b_{i}^{n}} \frac{\left(c_{i}\right)_{n}}{c_{i}^{n}} \frac{1}{\left(\frac{q}{b_{i}}\right)_{n}\left(\frac{q}{c_{i}}\right)_{n}} \\
=\frac{(q)_{N}\left(\frac{q}{b_{k} c_{k}}\right)_{N}}{\left(\frac{q}{b_{k}}\right)_{N}\left(\frac{q}{c_{k}}\right)_{N}} \sum_{n_{k} \geq n_{k-1} \geq \cdots \geq n_{1} \geq 0} \beta_{n_{1}} \frac{q^{n_{k}}\left(q^{-N}\right)_{n_{k}}\left(b_{k}\right)_{n_{k}}\left(c_{k}\right)_{n_{k}}}{\left(q^{-N} b_{k} c_{k}\right)_{n_{k}}} \\
\quad \times \prod_{i=1}^{k-1} \frac{q^{n_{i}} \frac{\left(b_{i}\right)_{n_{i}}}{b_{i}^{n_{i}}} \frac{\left(c_{i}\right)_{n_{i}}}{c_{i}^{n_{i}}\left(\frac{q}{b_{i} c_{i}}\right)_{n_{i+1}-n_{i}}}}{(q)_{n_{i+1}-n_{i}}\left(\frac{q}{b_{i}}\right)_{n_{i+1}}\left(\frac{q}{c_{i}}\right)_{n_{i+1}}} .
\end{aligned}
$$

A special Bailey pair is given by (see section 3.5 of $[\mathrm{An}]$ ):

$$
\begin{aligned}
& \alpha_{0}=1, \quad \alpha_{n}=(-1)^{n} q^{n(n-1) / 2}\left(1+q^{n}\right) \quad \text { for } n \geq 1, \\
& \beta_{0}=1, \quad \beta_{n}=0 \quad \text { for } n \geq 1 .
\end{aligned}
$$

Using the obvious limits

$$
\begin{aligned}
\lim _{c \rightarrow \infty} \frac{(c)_{n}}{c^{n}} & =(-1)^{n} q^{n(n-1) / 2}, \\
\lim _{c \rightarrow \infty}\left(\frac{q}{c}\right)_{n} & =1, \\
\lim _{c_{1}, c_{2} \rightarrow \infty} \frac{\left(c_{1}\right)_{n}\left(c_{2}\right)_{n}}{\left(q^{-N} c_{1} c_{2}\right)_{n}} & =(-1)^{n} q^{n(n-1) / 2} q^{N n},
\end{aligned}
$$

we see that the limit of the left hand side of (15), when $b_{i}, c_{i}$ tend to infinity, with $k$ replaced by $b$, is

$$
L H S=1+\sum_{n=1}^{N} \frac{q^{N n}\left(q^{-N}\right)_{n}}{\left(q^{N+1}\right)_{n}}\left(1+q^{n}\right) q^{b n^{2}} .
$$

Here the first term corresponds to $n=0$, and the sum of the second term terminates at $n=N$ since $\left(q^{-N}\right)_{n}=0$ if $n>N$. It is easy to check that

$$
\frac{q^{N n}\left(q^{-N}\right)_{n}}{\left(q^{N+1}\right)_{n}}\left(1+q^{n}\right)=\frac{(-1)^{n}}{\left[\begin{array}{c}
2 N \\
N
\end{array}\right]}\left[\begin{array}{c}
2 N \\
N-n
\end{array}\right]\left(q^{n / 2}+q^{-n / 2}\right) \text {. }
$$


Hence, with $N=k+1$, we have

$$
L H S=1+\frac{1}{\left[\begin{array}{c}
2 k+2 \\
k+1
\end{array}\right]} \sum_{n=1}^{k+1}(-1)^{n}\left[\begin{array}{c}
2 k+2 \\
k+1-n
\end{array}\right]\left(q^{n / 2}+q^{-n / 2}\right) q^{b n^{2}}
$$

Recall that

$$
\tilde{Y}(k, b)=\sum_{j=0}^{2 k+1}(-1)^{j}\left[\begin{array}{c}
2 k+1 \\
j
\end{array}\right] q^{b(j-k)^{2}} .
$$

The sum can be assumed from $j=-1$ to $j=2 k+1$, since the term of $j=-1$ is equal to 0 . Separating the case $j=k$ and combining any other $j$ with $2 k-j$, we have

$$
\tilde{Y}(k, b)=(-1)^{k}\left[\begin{array}{c}
2 k+1 \\
k
\end{array}\right]+\sum_{j=-1}^{k-1}(-1)^{j} q^{b(j-k)^{2}}\left(\left[\begin{array}{c}
2 k+1 \\
j
\end{array}\right]+\left[\begin{array}{c}
2 k+1 \\
2 k-j
\end{array}\right]\right) .
$$

It is easy to check that

$$
\left[\begin{array}{c}
2 k+1 \\
j
\end{array}\right]+\left[\begin{array}{c}
2 k+1 \\
2 k-j
\end{array}\right]=\frac{\{k+1\}}{\{2 k+2\}}\left[\begin{array}{c}
2 k+2 \\
j+1
\end{array}\right]\left(q^{(k-j) / 2}+q^{(j-k) / 2}\right) .
$$

Hence, using the new parameter $n=k-j$, we see that

$$
\begin{gathered}
\tilde{Y}(k, b)=(-1)^{k}\left[\begin{array}{c}
2 k+1 \\
k
\end{array}\right]+\frac{\{k+1\}}{\{2 k+2\}} \sum_{n=1}^{k+1}(-1)^{k-n} q^{b n^{2}}\left[\begin{array}{c}
2 k+2 \\
k+1-n
\end{array}\right]\left(q^{n / 2}+q^{-n / 2}\right) . \\
\operatorname{Using}\left[\begin{array}{c}
2 k+2 \\
k+1
\end{array}\right]=\left[\begin{array}{c}
2 k+1 \\
k
\end{array}\right] \frac{\{2 k+2\}}{\{k+1\}} \text {, from (16) we get } \\
\tilde{Y}(k, b)=(-1)^{k}\left[\begin{array}{c}
2 k+1 \\
k
\end{array}\right] \text { LHS. }
\end{gathered}
$$

The limit of the right hand side of (15), when $b_{i}, c_{i}$ tend to infinity, with $k$ replaced by $b$, is

$$
R H S=(q)_{N} \sum_{n_{b} \geq n_{b-1} \geq \cdots \geq n_{1}=0}(-1)^{n_{b}} q^{n_{b}\left(n_{b}-1\right) / 2+N n_{b}+n_{b}}\left(q^{-N}\right)_{n_{b}} \prod_{i=1}^{b-1} \frac{q^{n_{i}^{2}}}{(q)_{n_{i+1}-n_{i}}} .
$$

Note that $n_{1}=0$, since this is the only case when $\beta_{n_{1}} \neq 0$, and in the sum the index $n_{b} \leq N$, since $\left(q^{-N}\right)_{n_{b}}=0$ if $n_{b}>N$. An easy calculation shows that, with $k=N-1$,

$$
R H S=(-1)^{k+1}\{k+1\} ! H(k, b) .
$$

Since $L H S=R H S$, from (17) and (18) we get $\tilde{Y}(k, b)=-\frac{\{2 k+1\} !}{\{k\} !} H(k, b)$.

3.4.2. Proof of Proposition 3.1. Part (b), the difficult part, follows from Lemma 3.3 and Theorem 7 .

Now we prove part (a). Again noting that the two terms of the right hand side of (12) can be obtained from one another by the involution $n \rightarrow-n$, and using (14), we have

$$
\sum_{n}^{\xi}\{n\}\{n+k\} ! /\{n-k-1\} !=-2 \sum_{n}^{\xi}\left(\sum_{j=0}^{2 k+1}(-1)^{j}\left[\begin{array}{c}
2 k+1 \\
j
\end{array}\right] q^{n(j-k)}\right) .
$$


Note that $\sum_{n}^{\xi}\left(q^{n a}\right)=r$ or 0 , according to whether $a$ is divisible by $r$ or not. With $0 \leq j \leq 2 k+1 \leq r-2$, the only case with $j-k$ divisible by $r$ is when $j=k$. Hence

$$
\sum_{n}^{\xi}\{n\}\{n+k\} ! /\{n-k-1\} !=-2(-1)^{k} r \times \operatorname{ev}_{\xi}\left[\begin{array}{c}
2 k+1 \\
k
\end{array}\right] .
$$

Multiplying both sides by $\frac{\{k\} !}{\{2 k+1\} !}$ and using the well-known $r=\prod_{i=1}^{r-1}\left(1-\xi^{i}\right)$, we get part (a).

3.5. Proof of Proposition 3.2. Here we modify Ohtsuki's proof of a similar result [Oht1].

3.5.1. Linking pairing. A linking pairing on a finite abelian group $G$ is a nonsingular symmetric bilinear map from $G \times G$ to $\mathbb{Q} / \mathbb{Z}$. Two linking pairing $\nu, \nu^{\prime}$ on respectively $G, G^{\prime}$ are isomorphic if there is an isomorphism between $G$ and $G^{\prime}$ carrying $\nu$ to $\nu^{\prime}$. With the obvious block sum, the set of equivalence classes of linking pairings is a semigroup.

One type of linking pairing is given by non-singular square symmetric matrices with integer entries: any such $n \times n$ matrix $A$ gives rives to a linking pairing $\phi(A)$ on $G=\mathbb{Z}^{n} / A \mathbb{Z}^{n}$ defined by $\phi(A)\left(v, v^{\prime}\right)=v^{t} A^{-1} v^{\prime} \in \mathbb{Q} \bmod \mathbb{Z}$, where $v, v^{\prime} \in \mathbb{Z}^{n}$. If there is a diagonal matrix $A$ such that a linking pairing $\nu$ is isomorphic to $\phi(A)$, then we say that $\nu$ is of diagonal type.

Another type of pairing is the pairing $\phi_{b, a}$, with $a, b$ non-zero co-prime integers, defined on the cyclic group $\mathbb{Z} / b$ by $\phi_{b, a}(x, y)=a x y / b \bmod \mathbb{Z}$. It is clear that $\phi_{b, \pm 1}$ is also of the former type, namely, $\phi_{b, \pm 1}=\phi( \pm b)$, where $( \pm b)$ is considered as the $1 \times 1$ matrix with entry $\pm b$.

Proposition 3.5. Suppose $|G|=d$, with prime decomposition $d=\prod_{i=1}^{k} p_{i}^{e_{i}}$, and $\nu$ a linking pairing on $G$. There are pairs of non-zero, co-primes integers $\left(b_{j}, a_{j}\right), j=$ $1, \ldots, s$, such that each $b_{j}$ is a power of some $p_{i}$, and the block sum of $\nu$ and all the $\phi_{b_{j}, a_{j}}$ is of diagonal type.

Proof. The semigroup of linking pairings has the following generators in 3 groups; see $[\mathrm{KK}, \mathrm{Wa}]$ :

Group 1: $\phi\left( \pm p^{k}\right)$, where $p$ is a prime, and $k>0$.

Group 2: $\phi_{b, a}$ with $b=p^{k}$ as in Group 1, and $a$ is a non-quadratic residue modulo $p$ if $p$ is odd, or $a= \pm 3$ if $p=2$.

Group 3: $E_{0}^{k}$ on the group $\mathbb{Z} / 2^{k} \oplus \mathbb{Z} / 2^{k}$ with $k \geq 1$ and $E_{1}^{k}$ on the group $\mathbb{Z} / 2^{k} \oplus \mathbb{Z} / 2^{k}$ with $k \geq 2$.

For explicit formulas of $E_{0}^{k}$ and $E_{1}^{k}$; see $[\mathrm{KK}]$. We will use only a few relations between these generators, taken from [KK, Wa]. It's enough to prove the lemma when $\nu$ is one of the generators.

Any pairing in Group 1 is already of the form $\phi\left( \pm p^{k}\right)$.

Let $\nu=\phi_{b, a}$ be in Group 2. Suppose $p$ is odd; then one of the relations is $\phi_{b, a} \oplus \phi_{b, a}=\phi(b) \oplus \phi(b)$, which is of diagonal type. Suppose $b=2^{k}$; then $a= \pm 3$, and one of the relations says $\phi_{b, \pm 3} \oplus \phi_{b, \pm 3}=\phi(\mp b) \oplus \phi(\mp b)$.

Suppose $\nu=E_{0}^{k}$. One of the relations is $E_{0}^{k} \oplus \phi\left(-2^{k}\right)=\phi\left(2^{k}\right) \oplus \phi\left(-2^{k}\right) \oplus \phi\left(-2^{k}\right)$. Finally let $\nu=E_{1}^{k}$. One of the relations is $E_{1}^{k} \oplus \phi_{2^{k}, 3}=\phi\left(2^{k}\right) \oplus \phi\left(2^{k}\right) \oplus \phi\left(2^{k}\right)$. 
3.5.2. Linking pairing on the torsion group of $H_{1}(M, \mathbb{Z})$. Suppose $M$ is obtained from $S^{3}$ by surgery along a framed oriented link $L$, with a non-degenerate linking matrix $A$. Then $M$ is a rational homology 3 -sphere, and the linking pairing on $H_{1}(M, \mathbb{Z})$ is exactly $\phi(A)$. The following proposition was already implicitly given in $[\mathrm{Oht} 1]$.

Proposition 3.6. If the linking pairing of a rational homology 3-sphere $M$ is of diagonal type, then $M$ can be obtained from $S^{3}$ by surgery along an algebraically split link with integer framings.

Proof. Suppose $M$ is obtained from $S^{3}$ by surgery along a framed link $L$ with linking matrix $A$. By assumption, $\phi(A) \cong \phi(B)$, where $B$ is a diagonal matrix. In this case, it is known that there is a unimodular integral matrix $P$ such that $P^{t} A^{\prime} P=B^{\prime}$, where $A^{\prime}$ and $B^{\prime}$ are obtained from respectively $A$ and $B$ by blockadding a diagonal matrix with \pm 1 on the diagonal. Using the two Kirby moves on links, one can easily go from $L$ to another framed link with linking matrix $B^{\prime}$, which is diagonal. Surgery on the new link yields the same 3-manifold.

3.5.3. Proof of Proposition 3.2. Part (a). Suppose $H_{1}(M, \mathbb{Z})=\mathbb{Z}^{r} \oplus$ Tor, where Tor is the torsion part. Choose disjoint curves $\alpha_{1}, \ldots, \alpha_{r}$ in $M$ representing generators of the infinite part $\mathbb{Z}^{r}$ of $H_{1}(M, \mathbb{Z})$. By Poincare duality, there are oriented surfaces $S_{1}, \ldots, S_{r}$ in $M$ such that the algebraic intersection number between $\alpha_{i}$ and $S_{j}$ is $\delta_{i j}$. Using the standard tube construction if needed, we can assume that $S_{i}$ meets $\alpha_{i}$ at exactly 1 point, and misses all other $\alpha_{j}$.

Let $N\left(\alpha_{i}\right)$ be a small tubular neighborhood of $\alpha_{i}$ in $M$, and $S_{i}^{\prime}=S_{i} \backslash N\left(\alpha_{i}\right)$. Then $\beta_{i}:=\partial S_{i}^{\prime}$ is the intersection of $S_{i}$ with $\partial N\left(\alpha_{i}\right)$. Removing the interior of $N\left(\alpha_{i}\right)$ from $M$ and regluing back in such a way that the Dehn filling kills the homology class $\alpha_{i}$, we get a rational homology 3 -sphere $M^{\prime}$, together with $S_{i}^{\prime}$ in $M^{\prime}$. Each $S_{i}^{\prime}$ bounds the curve $\beta_{i}$ in $M^{\prime}$, such that in doing surgery along $\beta_{i}$ with framing 0 , from $M^{\prime}$ we get back $M$. Note that $H_{1}\left(M^{\prime}, \mathbb{Z}\right)=$ Tor.

If $N=L(b, a)$, the lens space, then the linking pairing on $H_{1}(N, \mathbb{Z})$ is exactly $\phi_{b, a}$. By Proposition 3.5, there are lens spaces $L\left(d_{i}, a_{i}\right)$, with properties as described in the statement of Proposition 3.2, such that the linking pairing of $M^{\prime \prime}:=M^{\prime} \#\left(\#_{i} L_{d_{i}, a_{i}}\right)$ is of diagonal type. By Proposition 3.6, $M^{\prime \prime}$ can be obtained from $S^{3}$ by integral surgery along a link $L^{\prime} \subset S^{3}$ with a diagonal linking matrix. Thus $S^{3} \backslash N\left(L^{\prime}\right)=M^{\prime \prime} \backslash N\left(L^{\prime \prime}\right)$, for a link $L^{\prime \prime}$ in $M^{\prime \prime}$. One can isotope $L^{\prime \prime}$ in $M^{\prime \prime}$ off the surfaces $S_{i}^{\prime}$, since the surface with non-trivial boundary can be isotoped to a lie in a small neighborhood of a graph. Thus all the surfaces $S_{i}^{\prime}$ can be considered as lying in $S^{3} \backslash L^{\prime}$. The link $L^{\prime} \cup\left\{\beta_{1}, \ldots, \beta_{r}\right\}$ has a diagonal linking matrix, with framing 0 on $\beta_{i}$. Doing surgery on $L^{\prime} \cup\left\{\beta_{1}, \ldots, \beta_{r}\right\}$ gives us $M^{\prime}$. This completes the proof of part (a). The proof of part (b) is totally similar.

\section{UNIVERSAL INVARIANT IN CYCLOTOMIC COMPLETION RINGS}

4.1. Proof of Theorem 3. First notice that if one can find an element $f \in \hat{\Lambda}_{d}$ such that

$$
\left(\frac{d}{r}\right) \tau_{M}(\xi)=\operatorname{ev}_{\xi}\left(q^{(1-d) / 4} f\right)
$$

then the injectivity of the map $\operatorname{ev}_{\Omega}$, with $\Omega=U_{d}$ in Theorem 4 , would show that $f$ is an invariant of $M$. The task now is to find such an $f$ for every rational homology 3 -sphere. Let us consider 3 cases. 
Case 1: $M=L(d, a)$, a lens space, with $d>0$. Let

$$
I_{M}:=q^{3 s(d, 1)-3 s(d, a)} \frac{1-q^{-1 / d}}{1-q^{-1}} .
$$

It is well-known that $3 d(s(d, 1)-s(d, a)) \in \mathbb{Z}$ (see $[\mathrm{RG}]$ ), hence $I_{M} \in \Lambda_{d}$. Using $s(d, 1)=(d-1)(d-2) / 12 d$ and $(3)$, one gets (19). Moreover, $I_{L(d, a)}$ is invertible in $\Lambda_{d}$.

Case 2: $M$ is obtained from $S^{3}$ by integral surgery along an algebraically split link $L$ as in section 3.2.1. We will use the notation of section 3.2.1. The following proposition will be proved later in this section.

Proposition 4.1. The element $\frac{Y(k, d)\{k\} !}{\{2 k+1\} !}$ is in $q^{(k+1)(k+2) / 4} \Lambda_{d}$.

Note that, by (11) and Theorem 7,

$$
\operatorname{sn}\left(b_{i}\right) \operatorname{ev}_{\xi}\left(Y\left(k_{i},-d_{i}\right) \frac{\left\{k_{i}\right\} !}{\left\{2 k_{i}+1\right\} !}\right)=H\left(k_{i},-b_{i}\right) .
$$

Let

$$
I_{M}:=q^{(d-1) / 4} \sum_{k_{i}=0}^{\infty} J_{L^{0}}\left(P_{k_{1}}^{\prime}, \ldots, P_{k_{m}}^{\prime}\right) \prod_{i=1}^{m} \operatorname{sn}\left(d_{i}\right) q^{\left(3 \operatorname{sn}\left(d_{i}\right)-d_{i}\right) / 4} Y\left(k_{i},-d_{i}\right) \frac{\left\{k_{i}\right\} !}{\left\{2 k_{i}+1\right\} !} .
$$

We will later prove the following.

Lemma 4.2. One has $I_{M} \in \hat{\Lambda}_{d}$.

By Habiro's theorem (Theorem 6) $J_{L^{0}}\left(P_{k_{1}}^{\prime}, \ldots, P_{k_{m}}^{\prime}\right)$ is divisible by $\frac{\{2 k+1\} !}{\{k\} !\{1\}}$. Hence by Lemma 2.1, if $k_{i}>(r-3) / 2$ for some $i$, then $\operatorname{ev}_{\xi}\left(J_{L^{0}}\left(P_{k_{1}}^{\prime}, \ldots, P_{k_{m}}^{\prime}\right)\right)=0$. Thus combining with (20) and (9), we get (19).

Case 3: $M$ is an arbitrary rational homology 3-sphere. By the diagonalizing lemma, there are lens spaces $M_{1}, \ldots, M_{l}$ such that $M^{\prime}=\left(\#_{i=1}^{l} M_{i}\right) \# M$ is of Case 2. Since each $I_{M_{j}}$ is invertible in $\Lambda_{d}$, we can define

$$
I_{M}=I_{M^{\prime}}\left(\prod_{i=1}^{l} I_{M_{i}}\right)^{-1}
$$

Using the product formula, we see that $I_{M}$ satisfies (19). This completes the proof of Theorem 3 .

4.2. Proof of Lemma 4.2. By Proposition 4.1, $Y\left(k_{i},-d_{i}\right) \frac{\left\{k_{i}\right\} !}{\left\{2 k_{i}+1\right\} !}$ is in $q^{\left(k_{i}+1\right)\left(k_{i}+2\right) / 4} \Lambda_{d}$, and by Theorem $6, J_{L^{0}}\left(P_{k_{1}}^{\prime}, \ldots, P_{k_{m}}^{\prime}\right)$ is divisible by $(q ; q)_{n}$. The only problem is, a priori, the term in the sum formula of $I_{M}$ might contain the fractional power $q^{1 / 2 d}$, and all we need to show is that we only need $q^{1 / d}$, but not $q^{1 / 2 d}$.

First, using Lemma 1.1 one sees that $J_{L^{0}}\left(P_{k_{1}}^{\prime}, \ldots, P_{k_{m}}^{\prime}\right) \prod_{i=1}^{m} \frac{q^{\operatorname{sn}\left(d_{i}\right) / 2}\left\{k_{i}\right\} !}{\left\{2 k_{i}+1\right\} !}$ is a rational function in $q$ (no fractional power of $q$ ). It suffices to prove that $q^{(d-1) / 4} \prod_{i=1}^{m} q^{\left(\operatorname{sn}\left(d_{i}\right)-d_{i}\right) / 4}$ is in $\mathbb{Z}\left[q^{ \pm 1 / d}\right]$. This is equivalent to the fact that

$$
\left.D:=d(d-1)-d \sum_{i=1}^{m}\left(\operatorname{sn}\left(d_{i}\right)-d_{i}\right)\right) \quad \text { is divisible by } 4 \text {. }
$$


We have $\operatorname{sn}\left(d_{i}\right)-d_{i}=\operatorname{sn}\left(d_{i}\right)\left(1-\left|d_{i}\right|\right)$. If the $\operatorname{sign} \operatorname{sn}\left(d_{i}\right)$ is changed, then $D$ is altered by $\pm 2 d\left(1-\left|d_{i}\right|\right)$, which is divisible by 4 , since $d_{i} \mid d$. Hence we can assume that every $\operatorname{sn}\left(d_{i}\right)=+1$.

We use induction on $m$. If $m=1$, then the statement is trivial. Note that

$$
\left(1-d_{1} d_{2}\right)-\left(1-d_{1}\right)-\left(1-d_{2}\right)=\left(1-d_{1}\right)\left(1-d_{2}\right),
$$

which, after multiplied by $d_{1} d_{2}$, is divisible by 4 . Hence the induction step can be carried out by replacing $d_{1}$ and $d_{2}$ with $d_{1}+d_{2}$.

4.3. Proof of Proposition 4.1. For every non-negative integer $n$ let $\mathcal{I}_{n}$ be the ideal in $\mathbb{Z}\left[t^{ \pm 1}, q^{ \pm 1}\right]$ generated by $c_{k, n}:=(t ; q)_{k}\left(q^{k+1}\right)_{n-k}, k=0,1, \ldots, n$. This ideal was used by Habiro in [Ha3]. Recall that $\tilde{\varphi}_{b}: \mathbb{Z}\left[t^{ \pm 1}, q^{ \pm 1}\right] \rightarrow \mathbb{Z}\left[q^{ \pm 1}\right]$ is the $\mathbb{Z}\left[q^{ \pm 1}\right]$-algebra homomorphism defined by $\tilde{\varphi}_{b}(t)=q^{b}$.

Proposition 4.3. Suppose $f(t, q) \in \mathbb{Z}\left[t^{ \pm 1}, q^{ \pm 1}\right]$. Then $\tilde{\varphi}_{b}(f)$ is divisible by $(q)_{n}$ for every integer $b$ if and only if $f \in \mathcal{I}_{n}$.

Proof. We use induction on $n$. Suppose the statement holds true for $n-1$. By induction,

$$
f=a_{0}(t, q) c_{0, n-1}+a_{1}(t, q) c_{1, n-1}+\cdots+a_{n-1}(t, q) c_{n-1, n-1} .
$$

Applying $\tilde{\varphi}_{0}$, noting that $\tilde{\varphi}_{0}\left(c_{k, n-1}\right)=0$ if $k>0$, one gets that $a_{0}(1, q)$ is divisible by $1-q^{n}$, or $a_{0}(t, q) \in(1-t)+\left(1-q^{n}\right)$. Note that $(1-t) c_{0, n-1}+\left(1-q^{n}\right) c_{0, n-1} \subset \mathcal{I}_{n}$. Hence the first term $a_{0}(t, q) c_{0, n-1}$ is in $\mathcal{I}_{n}$.

Similarly, successively consider $\tilde{\varphi}_{b}$ with $b=1,2, \ldots, n-1$; we see that each term $a_{i}(t, q) c_{i, n-1}$ is in $\mathcal{I}_{n}$.

Let $\frac{\mathcal{I}_{n}}{(q)_{n}}$ be the set of all rational functions of the form $f /(q)_{n}$, with $f \in \mathcal{I}_{n}$. Let $\psi_{d}: \mathbb{Z}\left[t^{ \pm 1}, q^{ \pm 1}\right] \rightarrow \mathbb{Z}\left[t^{ \pm 1}\right]$ be the $\mathbb{Z}\left[t^{ \pm 1}\right]$-algebra homomorphism defined by $\psi_{d}(q)=t^{d}$.

Lemma 4.4. For every non-negative integer $n$ one has $\psi_{d}\left(\frac{\mathcal{I}_{n}}{(q)_{n}}\right) \subset \Lambda_{d}$.

Proof. Fix a number $r$ co-prime with $d$. We need to show that the multiplicity of $\Phi_{r}$ in the prime decomposition of the denominator of

$$
\psi_{d}\left(\frac{c_{k, n}}{(q)_{n}}\right)=\frac{\prod_{i=0}^{k-1}\left(1-t^{i d+1}\right)}{\prod_{i=1}^{k}\left(1-t^{i d}\right)}
$$

is less than or equal to that in the numerator. Since $1-t^{n}=\prod_{m \mid n} \Phi_{m}$, we see that the multiplicity of $\Phi_{r}$ in the denominator is the number of elements of $\{d, 2 d, \ldots, k d\}$ which are divisible by $r$. Since $r$ and $d$ are co-prime, this is the number of elements of $\{1,2, \ldots, k\}$ which are divisible by $r$, and is equal to $\lfloor k / r\rfloor$.

The multiplicity of $\Phi_{r}$ in the numerator is the number of elements of $\{1,1+$ $d, \ldots, 1+(k-1) d\}$ which are divisible by $r$. This number is greater than or equal to $\lfloor k / r\rfloor$, since any $r$ consecutive elements of any progressive sequence contain one divisible by $r$.

Proof of Proposition 4.1. By Theorem 7, for every integer $b$,

$$
\tilde{\varphi}_{b} \frac{Z(k)(q)_{k}}{(q)_{2 k+1}} \in \mathbb{Z}\left[q^{ \pm 1}\right] .
$$


It follows from Proposition 4.3 that $Z(k)(q)_{k}$ belongs to $\mathcal{I}_{2 k+1}$, and hence

$$
Y(k, d) \frac{(q)_{k}}{(q)_{2 k+1}}=\psi_{d}\left(\frac{Z(k)(q)_{k}}{(q)_{2 k+1}}\right)
$$

belongs to $\Lambda_{d}$, by Lemma 4.4 . It remains to notice that for every $n$, the quotient $(q)_{n} /\{n\}$ ! is a power of $q^{1 / 2}$, and $\{2 k+1\} ! /\{k\}$ ! is in $q^{(k+1)(k+2) / 4} \mathbb{Z}[q]$.

\section{Cyclotomic completion}

After proving some additional facts in section 5.2 we will apply Habiro's result to our case.

5.1. Cyclotomic completion, general results. We fix a positive integer $d$. Recall that $\mathbb{N}_{d}$ is the set of all positive integers which are co-prime with $d$. Thus $\mathbb{N}:=\mathbb{N}_{1}$ is the set of all positive integers. Recall also $R_{d}=\mathbb{Z}[1 / d]$.

In this section we will identify $q^{1 / d}$ with $t$. Thus $A_{d}=R_{d}\left[t^{ \pm 1}\right]$, and $\Lambda_{d}$ is obtained from $A_{d}$ by inverting all the $\Phi_{n}(t), n \notin \mathbb{N}_{d}$. We also use $B_{d}:=R_{d}[t]$. One has $B_{d} \subset A_{d} \subset \Lambda_{d}$.

For a subset $S \subset \mathbb{N}$ let $\Phi_{S}^{*}$ be the multiplicative set in $B_{d}$ generated by $\Phi_{r}(t), r \in$ $S$. Then $\Phi_{S}^{*}$ is a directed set with respect to the divisibility relation $f \mid g$. For any ring $A$ containing $\mathbb{Z}[t]$, the principal ideals $(f)$ define a linear topology on $A$. Let

$$
A^{S}:=\lim _{f \in \Phi_{S}^{*}} A /(f)
$$

If $S^{\prime} \subset S$, then there is a natural algebra homomorphism $\rho_{S, S^{\prime}}: A^{S} \rightarrow A^{S^{\prime}}$. We want to know when $\rho_{S, S^{\prime}}$ is injective, for $A=B_{d}, A_{d}$, or $\Lambda_{d}$.

Proposition 5.1. (a) The map $\rho_{S, S \cap \mathbb{N}_{d}}: \Lambda_{d}^{S} \rightarrow \Lambda_{d}^{S \cap \mathbb{N}_{d}}$ is an isomorphism.

(b) With $t=q^{1 / d}, \Lambda_{d}^{\mathbb{N}}=\Lambda_{d}^{\mathbb{N}_{d}}$ is equal to $\hat{\Lambda}_{d}$.

(c) If $S \subset \mathbb{N}_{d}$, then the inclusions $B_{d} \hookrightarrow A_{d} \hookrightarrow \Lambda_{d}$ induce isomorphisms $B_{d}^{S} \cong$ $A_{d}^{S} \cong \Lambda_{d}^{S}$.

Remark 5.2. In particular $B_{d}^{\mathbb{N}_{d}}=\hat{\Lambda}_{d}$. Note that in the definition of $B_{d}^{\mathbb{N}_{d}}$, we don't have any denominator. The ring $B_{d}^{S}$ has been studied by Habiro [Ha2]. The author would like to thank Habiro for pointing out the isomorphism $B_{d}^{\mathbb{N}_{d}} \cong \hat{\Lambda}_{d}$, which simplifies the original version of the paper.

5.1.1. Injectivity of $\rho_{S, S^{\prime}}$. We say that $n, n^{\prime} \in \mathbb{N}$ are $p$-adjacent if $n / n^{\prime}=p^{e}$, where $p$ is a prime and $e$ an integer, and they are adjacent if they are $p$-adjacent for some prime $p$.

For $S^{\prime} \subset S \subset \mathbb{N}_{d}$ we write $S^{\prime} \prec S$ if for every $n \in S$ there are $n_{1}, \ldots, n_{k} \in S$ and $n_{k+1} \in S^{\prime}$, such that $n_{i}$ and $n_{i+1}$ are adjacent, and $n_{1}=n$.

For $m, n \in \mathbb{N}$ and any ring $R \supset \mathbb{Z}$, Habiro [Ha2] defined $m \Leftrightarrow_{R} n$ if $m, n$ are $p$-adjacent and $R$ is $p$-adically separated, i.e. $\bigcap_{j \geq 0} p^{j} R$ consists only of 0 . It is easy to see that if $p$ is a prime, $p \in \mathbb{N}_{d}$, then $R_{d}=\mathbb{Z}[1 / d]$ is $p$-separated. Hence if $m, n$ are in $\mathbb{N}_{d}$ and they are adjacent, then $m \Leftrightarrow_{R_{d}} n$ in Habiro's sense.

Suppose that $S_{0} \prec S \subset \mathbb{N}_{d}$. From theorem 4.2 of [Ha2] one has $\rho_{S, S_{0}}: B_{d}^{S} \rightarrow B_{d}^{S_{0}}$ is injective. Using Proposition 5.1, part (c) we get the following.

Theorem 8. Suppose that $S_{0} \prec S \subset \mathbb{N}_{d}$. Then $\rho_{S, S_{0}}: \Lambda_{d}^{S} \rightarrow \Lambda_{d}^{S_{0}}$ is injective. 
5.1.2. Evaluation map. Suppose $\xi$ is a root of order $r$, which belongs to $S \subset \mathbb{N}_{d}$. By Proposition 5.6 below, $\Lambda_{d} /\left(\Phi_{r}\right) \cong \mathbb{Z}[1 / d]\left[t^{ \pm 1}\right] /\left(\Phi_{r}\right)$. The last one is isomorphic to $\mathbb{Z}[1 / d][\xi]$ via $t \rightarrow \xi$. We can define the evaluation map ev $\tilde{v}_{\xi}: \Lambda_{d}^{S} \rightarrow \mathbb{Z}[1 / d][\xi]$ by the composition

$$
\Lambda_{d}^{S} \rightarrow \Lambda_{d}^{\left(\Phi_{r}\right)} \rightarrow \Lambda_{d} /\left(\Phi_{r}\right) \equiv \mathbb{Z}[1 / d][\xi] .
$$

Since $r$ and $d$ are co-prime, $\zeta:=\xi^{d}$ is also a root of order $r$, and by definition, $\tilde{\mathrm{ev}}_{\xi}(f)=\operatorname{ev}_{\zeta}(g)$, where $g$ is obtained from $f$ by the substitution $t \rightarrow q^{1 / d}$. Hence we get the following

Proposition 5.3. For $g \in \hat{\Lambda}_{d}$ and $\xi \in U_{d}$, one has $\operatorname{ev}_{\xi}(g) \in \mathbb{Z}[1 / d][\xi]$.

Suppose $\Omega$ is a set of roots of unity whose orders form a set $T$, which is a subset of $S \subset \mathbb{N}_{d}$. Using the evaluation at every element in $\Omega$ we can define

$$
\tilde{\mathrm{ev}}_{\Omega}: \Lambda_{d}^{S} \rightarrow \prod_{\xi \in \Omega} \mathbb{Z}[1 / d][\xi], \quad \tilde{\mathrm{ev}}_{\Omega}(f)=\left(\tilde{\operatorname{ev}}_{\xi}(f), \xi \in \Omega\right)
$$

Again using $B_{d}^{S} \cong \Lambda_{d}^{S}$, Theorem 6.1 of [Ha2] gives us the following.

Theorem 9. Suppose $\Omega, T, S$ are as above. Assume that there is $n \in S$ such that $\{n\} \prec S$ and there are infinitely many elements in $T$ adjacent to $n$. Then $\tilde{\mathrm{ev}}_{\Omega}: \Lambda_{d}^{S} \rightarrow \prod_{\xi \in \Omega} \mathbb{Z}[1 / d][\xi]$ is injective.

Theorem 4 is a special case of this theorem with $S=\mathbb{N}_{d}, n=1$.

5.2. Proof of Proposition 5.1. Part (a). Since $\Phi_{r}(t)$ is invertible in $\Lambda_{d}$ if $r$ is not in $\mathbb{N}_{d}$, we have $\Lambda_{d}^{S}=\Lambda_{d}^{S \cap \mathbb{N}_{d}}$.

Part (b). Since $1-t^{n}=\prod_{r \mid n} \Phi_{r}(t)$, one has that $\frac{1-t^{n d}}{1-t^{n}}$ is the product of several $\Phi_{r}$ with $r \notin \mathbb{N}_{d}$. Hence $\frac{1-t^{n d}}{1-t^{n}}$ is invertible in $\Lambda_{d}$. This means if $q=t^{d}$, then the elements $(t ; t)_{n}$ and $(q ; q)_{n}$ defines the same principal ideal in $\Lambda_{d}$, and hence they define the same completion, or $\Lambda_{d}^{\mathbb{N}}=\hat{\Lambda}_{d}$.

The rest of this subsection is devoted to a proof of part (c).

Lemma 5.4. (a) If $m, n$ are not adjacent, then $\left(\Phi_{m}\right)+\left(\Phi_{n}\right)=(1)$ in $\mathbb{Z}[t]$. $\mathbb{Z}[t]$.

(b) If $m=n p^{e}$, where $e>0$ and $p$ a prime, then $\left(\Phi_{m}\right)+\left(\Phi_{n}\right)=(p)+\left(\Phi_{n}\right)$ in

Proof. (a) is a well-known fact.

(b) Let $a=t^{n p^{e-1}}$; then $\Phi_{m}$ is a divisor of

$$
g(t)=\frac{1-a^{p}}{1-a}=1+a+\cdots+a^{p-1} .
$$

Since $a(\xi)=1$, and hence $g(\xi)=p$ if $\xi$ is a root of unity of order $n$, we have that $g(t)=p\left(\bmod \Phi_{n}\right)$. This means $\Phi_{m}$ divides $p$ in $\mathbb{Z}[t] /\left(\Phi_{n}\right)$. It is known that there is a positive $k$ such that $\Phi_{m}=\Phi_{n}^{k}(\bmod p)$. It follows that $p$ divides $\Phi_{m}$ in $\mathbb{Z}[t] /\left(\Phi_{n}\right)$. Thus in $\mathbb{Z}[t] /\left(\Phi_{n}\right), \Phi_{m}$ and $p$ define the same principal ideal. This is equivalent to $\left(\Phi_{m}\right)+\left(\Phi_{n}\right)=(p)+\left(\Phi_{n}\right)$ in $\mathbb{Z}[t]$.

Corollary 5.5. Suppose $m_{1}, \ldots, m_{k}$ are co-prime with $d$ and $n$ is not, i.e. $m_{1}, \ldots$, $m_{k} \in \mathbb{N}_{d}$ and $n \notin \mathbb{N}_{d}$. Then $\Phi_{n}$ is invertible in $\mathbb{Z}[1 / d][t] /(f)$, where $f=\prod_{i=1}^{k} \Phi_{m_{i}}$. Here $m_{1}, \ldots, m_{k}$ are not necessarily distinct. 
Proof. Note that in a commutative ring $A$, an element $a$ is invertible in $A /(b)$ if and only if $(a)+(b)=(1)$. If $(a)+(b)=(1)$ and $(a)+(c)=(1)$, then, multiplying together, one gets $(a)+(b c)=(1)$. Hence it's enough to consider the case $k=1$, with $m_{1}=m$.

If $m, n$ are not adjacent, then $\left(\Phi_{n}\right)+\left(\Phi_{r}\right)=(1)$ in $\mathbb{Z}[t]$, hence we are done in this case.

Suppose $n / m=p^{e}$, with $p$ a prime. Then $e>0$, since otherwise $n$ is a divisor of $m$ and hence is co-prime with $d$. By the same reason, $p$ is a divisor of $d$. By Lemma $5.4,\left(\Phi_{m}\right)+\left(\Phi_{n}\right) \supset(p)$ in $\mathbb{Z}[t]$. Since $p$ divides $d$, it is invertible in $\mathbb{Z}[1 / d][t]$, hence $\left(\Phi_{m}\right)+\left(\Phi_{n}\right)=(1)$ in $\mathbb{Z}[1 / d][t]$.

Proposition 5.6. Suppose $f=\prod_{i=1}^{k} \Phi_{m_{i}}$ with $m_{i} \in \mathbb{N}_{d}$. Then the homomorphism $A_{d} /(f) \rightarrow \Lambda_{d} /(f)$, induced from the embedding $A_{d} \hookrightarrow \Lambda_{d}$, is an isomorphism.

Proof. Recall that $\Lambda_{d}$ is obtained from $A_{d}$ by inverting all the $\Phi_{n}, n \notin \mathbb{N}_{d}$.

By Corollary 5.5, every $\Phi_{n}, n \notin \mathbb{N}_{d}$, is invertible in $A_{d} /(f)$. This proves the surjectivity. Injectivity follows easily from the fact that every $\Phi_{n}, n \notin \mathbb{N}_{d}$, is coprime with $f$.

Corollary 5.7. If $S \subset \mathbb{N}_{d}$, then the inclusion $A_{d} \hookrightarrow \Lambda_{d}$ induces an isomorphism $A_{d}^{S} \cong \Lambda_{d}^{S}$.

Proposition 5.8. For any $S \subset \mathbb{N}$, the inclusion $B_{d} \hookrightarrow A_{d}$ induces an isomorphism $B_{d}^{S} \cong A_{d}^{S}$.

Proof. Note that $\Phi_{n}(0)= \pm 1$; one has $(t)+\left(\Phi_{n}(t)\right)=(1)$ in $\mathbb{Z}[t]$. It follows that if $f$ is the product of several $\Phi_{n}(t)$, then $t$ is invertible in $\mathbb{Z}[t] /(f)$, and hence in $B_{d}[t] /(f)$. It follows that $B_{d}^{S} \cong A_{d}^{S}$.

Part (c) of Proposition 5.1 follows from Corollary 5.7 and Proposition 5.8.

5.3. Proof of Theorem 5. Part (a). Using Proposition 5.1, with $t=q^{1 / d}$, we can identify $\hat{\Lambda}_{d}$ with $\Lambda_{d}^{\mathbb{N}_{d}}$. The Taylor expansion map $T_{1}$ is then the map $\rho_{\mathbb{N}_{d},\{1\}}$ : $\Lambda_{d}^{\mathbb{N}_{d}} \rightarrow \Lambda_{d}^{\{1\}}$. The image space $\Lambda_{d}^{\{1\}}$ is equal to $\mathbb{Z}[1 / d]\left[t^{ \pm 1}\right]^{\left(\Phi_{1}\right)}=\mathbb{Z}[1 / d][[t-1]]$, by Proposition 5.6.

As explained, to convert a power series in $t-1=q^{1 / d}-1$ into a power series in $q-1$, one use

$$
q^{1 / d}-1=(1+(q-1))^{1 / d}-1=\sum_{n=1}^{\infty}\left(\begin{array}{c}
1 / d \\
n
\end{array}\right)(q-1)^{n} .
$$

It is well-known that $\left(\begin{array}{c}1 / d \\ n\end{array}\right) \in \mathbb{Z}[1 / d]$ for every $n$. Hence the image of $T_{1}$ is in $\mathbb{Z}[1 / d][[q-1]]$.

Part (b), the most difficult part, is a special case of Theorem 8 , with $S=\mathbb{N}_{d}$ and $S^{\prime}=\{1\}$.

Part (c). First let us recall the Ohtsuki series, which is, in a sense, a numbertheoretical expansion around $q=1$ of a function $f(q)$ with domain $U_{d}$ (for some $d$ ) such that $f(\xi) \in \mathbb{Z}[\xi]$.

Fix an odd prime $r$, and let $\xi_{r}=\exp (2 \pi i / r)$. Since $f\left(\xi_{r}\right) \in \mathbb{Z}\left[\xi_{r}\right]$, which is a free $\mathbb{Z}$-module spanned by $\left(\xi_{r}-1\right)^{j}, j=0,1, \ldots, r-2$, there are integers $a_{r, j}$ such that

$$
f\left(\xi_{r}\right)=\sum_{j=0}^{r-2} a_{r, j}(\xi-1)^{j},
$$


where $a_{r, j}$ are integers. In general $a_{r, j}$ depends on $r$. If there are rational numbers $\lambda_{j}$ such that for big enough prime $r$ one has $\lambda_{j} \equiv a_{r, j}(\bmod r)$, then the series $\tilde{T}(f):=\sum_{j=0}^{\infty} \lambda_{j}(\xi-1)^{j}$ is called the Ohtsuki series of $f$. (It easy to see that such a series, if it exists, is unique.)

The Ohtsuki series of $M$ is the Ohtsuki series of the function $f$ defined by $f\left(\xi_{r}\right)=\left(\frac{d}{r}\right) \tau_{M}\left(\xi_{r}\right)$. The existence of $\tilde{T}(f)$ was established by Ohstuki for $s l_{2}$; for general Lie algebras it was established by the author in [Le3].

The following facts are easy to verify:

(1) If $\tilde{T}(f), \tilde{T}(g)$ exist, then so does $\tilde{T}(f g)$, and $\tilde{T}(f g)=\tilde{T}(f) \tilde{T}(g)$.

(2) For $f \in \hat{\Lambda}_{d}, \tilde{T}(f)$ exists, and $\tilde{T}(f)=T_{1}(f)$.

(3) For $f(\xi):=\operatorname{ev}_{\xi}\left(q^{(1-d) / 4}\right), \tilde{T}(f)$ exists, and $\tilde{T}(f)=T_{1}(f)$.

Since $\left(\frac{d}{r}\right) \tau_{M}(\xi)=\operatorname{ev}_{\xi}\left(q^{(1-d) / 4} I_{M}\right)$ and $I_{M} \in \hat{\Lambda}_{d}$, part (c) follows immediately from the above facts.

\section{ACKNOWLEDGEMENT}

The author wishes to thank the anonymous referee, A. Beliakova, S. Garoufalidis, T. Ohtsuki and especially K. Habiro for valuable comments. A great deal of this paper was inspired by Habiro's work.

\section{REFERENCES}

[An] G. Andrews, q-series: their development and applications in analysis, number theory, combinatorics, physics, and computer algebra, regional conference series in mathematics, number 66, Amer. Math. Soc., 1985. MR858826 (88b:11063)

[BBL] A. Beliakova, C. Blanchet, and T. T. Q. Le, Laplace transform and universal sl(2) invariants, preprint math.QA/0509394.

[GM] P. Gilmer and G. Masbaum, Integral lattices in TQFTs, preprint math.QA/0411029.

[Ha1] K. Habiro, On the quantum $\mathfrak{s l}_{2}$ invariants of knots and integral homology spheres, Geom. Topol. Monogr. 4 (2002) 55-68. MR2002603 (2004g:57023)

[Ha2] K. Habiro, Cyclotomic completions of polynomial rings, Publ. Res. Inst. Math. Sci. 40 (2004), 1127-1146. MR2105705 (2005j:13010)

[Ha3] K. Habiro, An integral form of the quantized enveloping algebra of $\mathrm{sl}_{2}$ and its completions, J. Pure Appl. Algebra 211 (2007), 265-292. MR2333771

[HL] K. Habiro and T. T. Q. Lê, in preparation.

[KM] R. Kirby and P. Melvin, The 3-manifold invariants of Witten and Reshetikhin-Turaev for sl(2,C), Invent. Math. 105 (1991), 473-545. MR1117149 (92e:57011)

[KK] A. Kawauchi and S. Kojima, Algebraic classification of linking pairings on 3-manifolds, Math. Ann. 253 (1980), 29-42. MR594531 (82b:57007)

[Jo] V. F. R. Jones, Hecke algebra representations of braid groups and link polynomials, Ann. of Math. (2) 126 (1987), 335-388. MR908150 (89c:46092)

[Le1] T. T. Q. Lê, An invariant of integral homology 3-spheres which is universal for all finite type invariants, AMS translation series 2, Eds. V. Buchtaber and S. Novikov, 179 (1997), 75-100. MR1437158 (99m:57008)

[Le2] T. T. Q. Lê, Integrality and symmetry of quantum link invariants, Duke Math. J. 102 (2000) 273-306. MR1749439 (2001c:57015)

[Le3] T. T. Q. Lê, Quantum invariants of 3-manifolds: integrality, splitting, and perturbative expansion, Topology Appl. 127 (2003), 125-152. MR1953323 (2005b:57026)

[LL] B.-H. Li and T.-J. Li, Generalized Gaussian sums: Chern-Simons-Witten-Jones invariants of lens-spaces, J. Knot Theory Ramifications 5 (1996), 183-224. MR1395779 (97e:57018)

[Ma] G. Masbaum, Skein-theoretical derivation of some formulas of Habiro, Algebraic and Geometric Topology 3 (2003), 537-556. MR1997328 (2004f:57013)

[MR] G. Masbaum and J. Roberts, A simple proof of integrality of quantum invariants at prime roots of unity, Math. Proc. Camb. Phil. Soc. 121 (1997), 443-454. MR1434653 (98h:57037) 
[MW] G. Masbaum and H. Wenzl, Integral modular categories and integrality of quantum invariants at roots of unity of prime order, J. Reine Angew. Math. 505 (1998), 209-235. MR1662260 (2000a:57043)

[Mu] H. Murakami, Quantum SO(3)-invariants dominate the SU(2)-invariant of Casson and Walker, Math. Proc. Camb. Phil. Soc. 117 (1995), 237-249. MR1307078 (95k:57027)

[LMO] T. T. Q. Lê, J. Murakami, and T. Ohtsuki, On a universal perturbative invariant of 3-manifolds, Topology 37 (1998), 539-574. MR1604883 (99d:57004)

[Oht1] T. Ohtsuki, A polynomial invariant of rational homology 3-spheres, Invent. Math. 123 (1996), 241-257. MR1374199 (97i:57018)

[Oht2] T. Ohtsuki, Quantum invariants. A study of knots, 3-manifolds, and their sets, Series on Knots and Everything, 29, World Scientific Publishing Co., Inc., River Edge, NJ, 2002. MR1881401 (2003f:57027)

[RG] H. Rademacher and E. Grosswald, Dedekind Sums, Amer. Math. Soc., Washington D. C., 1972. MR0357299 (50:9767)

[Ros] L. Rozansky, On p-adic properties of the Witten-Reshetikhin-Turaev invariant, preprint math.QA/9806075, 1998.

[TY] T. Takata and Y. Yokota, The PSU $(n)$ invariants of 3-manifolds are polynomials, J. Knot Theory Ramifications 8 (1999), no. 4, 521-532. MR1697388 (2000k:57016)

[Tur] V. G. Turaev, Quantum invariants of knots and 3-manifolds, de Gruyter Studies in Mathematics 18, Walter de Gruyter, Berlin, New York, 1994. MR1292673 (95k:57014)

[Wa] C. T. C. Wall, Quadratic forms on finite groups, and related topics, Topology 2 1963, 281-298 MR0156890 (28:133)

Department of Mathematics, Georgia Institute of Technology, Atlanta, Georgia 30332-0160

E-mail address: letu@math.gatech.edu 